\title{
El debate sobre el estado de la nación como parte del ritual democrático-parlamentario: análisis del discurso de Rajoy y Rubalcaba en 2014
}

\author{
Dimitrina Jivkova Semova \\ Universidad Complutense de Madrid \\ d.jivkova@ccinf.ucm.es \\ Graciela Padilla Castillo \\ Universidad Complutense de Madrid \\ gracielp@ucm.es \\ Noé PERnía PeñalVer \\ Universidad Complutense de Madrid \\ noepernia@gmail.com
}

Recibido: 27 de diciembre de 2014

Aceptado: 15 de septiembre de 2015

\section{Resumen}

En este artículo, analizamos el Debate sobre el Estado de la Nación del año 2014. La metodología aplicada consiste en la codificación de los discursos del presidente del gobierno, Mariano Rajoy (Partido Popular) y el entonces líder de la oposición, Alfredo Pérez Rubalcaba (Partido Socialista Obrero Español), extrayendo nubes de palabras, mapas ramificados y árboles de palabras que han mostrado los conceptos y premisas más frecuentes. Este análisis previo de doble dimensión, cuantitativa y cualitativa, hace mucho más fácil y viable el posterior análisis del discurso donde nos centramos en los distintos tipos de argumentos en el acto comunicativo: demanda/solución, premisas circunstanciales, premisasmetas, premisas de valor, premisas de recursos y metas, opciones alternativas/abordar opciones alternativas.

Palabras clave: Debate sobre el estado de la nación, análisis del discurso, Fairclough, crisis, economía.

The State of the Nation Debate as part of the democratic parliamentary ritual: Rajoy and Rubalcaba discourse analysis, 2014

\begin{abstract}
In this article we analyze the Debate on the State of the Nation 2014. The methodology consists in coding the speeches of the prime minister, Mariano Rajoy (PP) and the then opposition leader Alfredo Perez Rubalcaba (PSOE) through extracting word clouds, branched maps and word trees that have shown the most common concepts and premises. This preliminary analysis of two dimensions, quantitative and qualitative, makes it much easier and viable subsequent discourse analysis where we focus on the different types of arguments in the communicative act: claim/solution, circumstantial premises, goal premises, value premises, means-goal premises, alternative options/addressing alternative options.
\end{abstract}

Keywords: Debate on the state of the nation, discourse analysis, Fairclough, crisis, economy.

\section{Referencia normalizada}

JIVKOVA SEMOVA, Dimitrina; PADILLA CASTILLO, Graciela; y PERNÍA PEÑALVER, Noé (2016): "El debate sobre el estado de la nación como parte del ritual democrático-parlamentario: análisis del discurso de Rajoy y Rubalcaba en 2014”. Estudios sobre el Mensaje Periodístico. Vol. 22, Núm. 1 (enerojunio), págs.: 375-390. Madrid, Ediciones Complutense.

Sumario: 1. Introducción y objetivos. 2. Fuentes y metodología. 3. Desarrollo; 3.1. Frecuencias; 3.2. Nodo "crisis"; 3.3. Nodo "empleo"; 3.4. Nodo "economía"; 3.5. Nodo "social"; 4. Conclusiones. 5. Referencias bibliográficas. 


\section{Introducción y objetivos}

El debate sobre el estado de la nación se celebra una vez al año, en el Congreso de los Diputados de España, y aborda, durante una o dos jornadas, toda la política general llevada a cabo por el Gobierno, durante el último año. No aparece como celebración o evento obligatorio en la Constitución Española, pero se ha establecido como una práctica parlamentaria anual ineludible desde el año 1983. Fue entonces cuando el presidente de Gobierno, Felipe González, propuso "un debate sobre la situación general de España". Desde allí, se ha celebrado en 24 ocasiones, hasta 2014, y sólo dejó de tener lugar cuando coincidió con la convocatoria de elecciones generales. Durante los gobiernos de Felipe González (PSOE), se celebraron diez debates sobre el estado de la nación; durante los gobiernos de José María Aznar (PP), seis; durante los gobiernos de José Luis Rodríguez Zapatero (PSOE), otros seis; y durante el gobierno de Mariano Rajoy (PP), otros dos, por el momento.

Aunque no exista regulación específica, como acabamos de indicar, siempre tiene la misma estructura: el presidente del Gobierno abre el debate y le siguen los distintos representantes de los demás partidos políticos de la Cámara, en orden jerárquico, de más a menos escaños o representación. A pesar de ese orden, todos los grupos políticos disponen, obligatoriamente, del mismo tiempo para hacer su intervención. Después, el presidente de Gobierno toma la palabra y puede responder a todas las intervenciones anteriores, a la vez o una a una. Finalmente, los grupos tienen un último turno de réplica como intervención final.

Una vez acaba el debate sobre el estado de la nación, se presentan las propuestas de resolución de los grupos parlamentarios que han intervenido y éstas son debatidas y votadas en una sesión parlamentaria posterior. Desde el año 2005, y atendiendo la propuesta del Gobierno de entonces, se optó por separar el debate de la votación de esas propuestas posteriores.

Por imperativo legal, el ente público de Radio Televisión Española (RTVE) emite en directo el debate completo, sin anuncios y en abierto para todo el territorio nacional, por un canal de radio y por otro canal de televisión, además de su página Web, www.rtve.es. Cuenta con retransmisión especial para discapacitados visuales y auditivos. Radio 5 y Canal 24 Horas son las cadenas públicas encargadas de su retransmisión, en los últimos años. Además, cualquier otra emisora de radio o televisión tiene la posibilidad de emitirlo al mismo tiempo, total o parcialmente, según decida.

El debate sobre el estado de la nación del año 2014 se desarrolló durante dos jornadas: el martes 25 de febrero y el miércoles 26 de febrero. A las 10:30 de la mañana, del primer día, el presidente Mariano Rajoy (PP, Partido Popular) comenzó a abordar su segundo debate como presidente. Cataluña, la lucha contra el terrorismo y ETA, el aborto o el paro eran algunos de los asuntos más esperados de la discusión. Por la tarde, Alfredo Pérez Rubalcaba, a las 16:05 horas, comenzó su intervención como líder del principal partido de la oposición (PSOE, Partido Socialista Obrero Español). Le siguieron Durán i Lleida (CIU, Convergència i Unió), Cayo Lara (Grupo Izquierda Plural), Joan Coscubiela (ICV, Iniciativa per Catalunya Verds), Chesús Yuste (Chunta Aragonesista) y Rosa Díez (UPyD, Unión Progreso y Democracia). 
Durante la segunda jornada, del 26 de febrero de 2014, intervinieron Mikel Errekondo (Amaiur), Alfred Bosch i Pascual (ERC, Esquerra Republicana de Catalunya), Olaia Fernández (BNG, Bloque Nacionalista Galego), Joan Baldoví (CompromísEquo), Carlos Salvador (UPN, Unión del Pueblo Navarro), Alfonso Alonso, portavoz del PP, y de nuevo, Mariano Rajoy, como presidente del Gobierno. En este artículo, abordamos y comparamos solamente los discursos de Rajoy y Rubalcaba, pero deseamos que sirva de prospectiva académica de investigación para analizar los discursos de los demás líderes y partidos, en este debate o en cualquier otro.

Este artículo analiza los principales temas y palabras del debate sobre el estado de la nación 2014, en los discursos del presidente de Gobierno, Mariano Rajoy (Partido Popular), y el entonces líder de la oposición, Alfredo Pérez Rubalcaba (Partido Socialista Obrero Español). Hemos codificado los discursos de los dos dirigentes en dos pasos. En primer lugar, hemos procedido a la extracción de las nubes de palabras, mapas ramificados y árboles de palabras para ver los conceptos más frecuentes en los materiales seleccionados. Para ello, hemos utilizado el software NVivo, un programa diseñado para el análisis de datos cualitativos, que facilita la codificación y análisis de documentos de trabajo. Mediante la creación de nodos y su correspondiente codificación, hemos conseguido categorizar las ideas y, también, organizar las mismas por conceptos previamente elegidos: "Crisis", "Economía", "Empleo" y "Social".

En segundo lugar, hemos analizado el discurso desde el planteamiento propuesto por Isabela y Norman Fairclough (2012), que nace a raíz del análisis del discurso del Partido Liberal y del Primer Ministro, Tony Blair. Asimismo, hemos tomado como referentes teóricos cuatro autores que continúan la teoría de Norman Fairclough, como son Blommaert y Bulcaen (2000), Hidalgo Tenorio (2011) y Dunmire (2012).

\section{Fuentes y metodología}

La primera parte del análisis ha sido llevada a cabo con la ayuda del software NVivo, un programa diseñado para el análisis de datos cualitativos, que facilita la codificación y análisis de documentos de trabajo. Permite almacenar, organizar y obtener informes resumidos de los datos más significativos que emergen del análisis. Nvivo, como herramienta, nos ha permitido combinar una doble dimensión integrando la perspectiva narrativa (más cualitativa) y analítica (más cuantitativa). En NVivo, los contenedores para almacenar temas o ideas se llaman "nodos" y pueden representar cualquier objeto/idea: personas, lugares y organizaciones a los que se puede asignar datos o atributos de distinta índole.

El primer paso consiste en la codificación de los textos y su posterior comprobación. Después de esta fase inicial, hemos procedido a la extracción de las nubes de palabras, mapas ramificados y árboles de palabras para ver los conceptos más frecuentes en los materiales seleccionados (textos íntegros del discurso de Rajoy y Rubalcaba en el debate sobre el estado de la nación), con el fin de explorar el contexto que rodea las palabras. Mediante la creación de nodos libres y su correspondiente codificación, hemos conseguido categorizar las ideas y, también, organizar las mismas por conceptos previamente elegidos ("Crisis", "Economía", "Empleo" y "Social"). 
Este análisis previo de doble dimensión, cuantitativa y cualitativa, hace mucho más fácil y viable el posterior análisis del discurso, donde nos centramos en el planteamiento propuesto por Isabela y Norman Fairclough (2012: 88-89), que nace a raíz del análisis del discurso del Partido Liberal y del Primer Ministro, Tony Blair. Fairclough y Fairclough (2012: 88-89) proponen clasificar los argumentos en el acto comunicativo de la siguiente manera: Demanda/solución (D), Premisas Circunstanciales /problemas (PC), Premisas-Metas (PM), Premisas de Valor (PV), Premisas de Medios/recursos y metas (PMM), Opciones Alternativas (OA), Abordar las Opciones Alternativas (AOA).

Según los dos autores, como mínimo, la reconstrucción más sucinta de un argumento debe incluir: Premisas Circunstanciales/problemas (PC), Premisas-Metas (PM), Premisas de Valor (PV) y Demanda/solución (D). Asimismo, definen y sistematizan todos los elementos, de la siguiente forma, en el discurso de Tony Blair (Fairclough y Fairclough, 2012: 88-89):

- Demanda/solución (D): "Debemos analizar el desafío del cambio y afrontarlo / prepararnos / y adoptar la política de la tercera vía.

- Premisas Circunstanciales/problemas (PC): "Éste es un mundo de cambios dramáticos (empírico); el cambio está arrasando el mundo; el cambio es un desafío para el mundo moderno (compromisos implícitos para garantizar la equidad, la prosperidad, sirviendo a los intereses de toda la nación, en nombre del gobierno).

- Premisas-Metas (PM): "Nuestra meta es sobrevivir y prosperar, hacer Gran Bretaña más competitiva y mejor en la generación de riqueza" (Meta).

- Premisas de Valor (PV): "Una preocupación real por las necesidades de la nación, la prosperidad y la justicia".

- Premisas de Medios/recursos y metas (PMM): "La única manera (de alcanzar estas metas) es analizando y afrontando el desafío del cambio".

Atendiendo a Fairclough y Fairclough (2012: 89), la deliberación también debe incluir, como mínimo, qué razones apoyarían no llevar a cabo las acciones propuestas y otras opciones alternativas. Se examinan todas las opciones, analizando los pros y contras de cada propuesta. La deliberación es un modelo normativo, un género en sí mismo, y evaluar un argumento real no implica, por supuesto, que los argumentos particulares sean los mejores ejemplos de la deliberación. De hecho, se pueden incluir los posibles impactos negativos de la propuesta y hacer un retrato más real y completo de todo el argumentario general (Fairclough y Fairclough, 2012: 140).

Para la metodología del análisis del discurso, propuesto en este artículo, también hemos incluido otras tres fuentes: Blommaert y Bulcaen (2000), Hidalgo Tenorio (2011) y Dunmire (2012). Jan Blommaert y Chris Bulcaen (2000: 447-446) proponen un estudio actualizado del Critical Discourse Analysis (CDA) o Análisis Crítico del Discurso, ocupándose de las relaciones de poder y desigualdad del lenguaje. Comentan las teorías de Norman Fairclough, al que citan como uno de los académicos más reputados en este asunto, junto con otras reacciones críticas y nuevos paradigmas y disciplinas orientados al estudio del lenguaje. 
Explican que el Análisis Crítico del Discurso surgió a finales de la década de 1980, como un desarrollo en los estudios del discurso europeos, encabezados por Norman Fairclough, Ruth Wodak o Teun van Dijk, entre otros. Desde entonces, según Blommaert y Bulcaen (2000: 447), se ha convertido en una de las ramas más influyentes y visibles del análisis del discurso. Recuerdan que Fairclough esbozó un marco tridimensional para concebir el análisis del discurso: la dimensión del discurso como texto, la dimensión del discurso como práctica discursiva y la dimensión del discurso como práctica social. A partir de esta tercera dimensión, Fairclough construyó su enfoque principal: las hegemonías cambian según cambian los discursos y la manera en que un discurso puede ser re-presentado, re-pronunciado y re-escrito, arroja luz sobre la aparición de un nuevo orden del discurso, luchas por la normatividad, intentos de control y resistencia contra los regímenes de poder (Blommaert y Bulcaen, 2000: 449).

Tras un profundo y exhaustivo estudio teórico del Critical Discourse Analysis (CDA) o Análisis Crítico del Discurso, proponen un nuevo paradigma crítico, basado en tres elementos: Ideología, Desigualdad y poder, y Teoría Social (Blommaert y Bulcaen, 2000: 456). En Ideología, citan nuevas investigaciones sobre la mediación, la intertextualidad y la representación, atendiendo a autores como Peirce, Bajtin, y Habermas, que dieron lugar a importantes conocimientos sobre la autoridad y las jerarquías. En Desigualdad y poder, comentan los trabajos de Bloch, Brennes y Myers, Durante, Mei o Flowerdeu, para remarcar cómo el asunto de la desigualdad y del posicionamiento individual y de los grupos ha ganado posiciones en la investigación social y política. Concretamente, citan a Charles Briggs, que mostró cómo la construcción de textos y discursos podía dar lugar a potentes efectos sociales, centrándose así en las desigualdades en el control de los contextos y en los géneros específicos o formas de hablar. El poder depende no sólo del acceso a los recursos, sino también del acceso a los contextos en los que se pueden utilizar esos recursos (Blommaert y Bulcaen, 2000: 458).

Por último, en cuanto a la Teoría Social, recuerdan que el lenguaje se estudió por lo que dice acerca de la sociedad y que el método lingüístico debería estar abierto a ideas teóricas sobre la estructura de las sociedades (Blommaert y Bulcaen, 2000: 459). Interpretan que las fuentes de nuevas ideas son infinitas y que se ha hecho poco uso del gran número de ideas potencialmente útiles de otras disciplinas. Exponen que, históricamente, la Teoría Social apenas se ha utilizado como un recurso para los estudios críticos del lenguaje, a pesar de las relevantes contribuciones de Marc Bloch, Fernand Braudel, Carlo Ginzburg, Peter Burke, Immanuel Wallerstein y Edward Thompson (Blommaert y Bulcaen, 2000: 460). Igualmente, proponen rescatar la potencialidad de nuevas reinterpretaciones etnográficas, establecidas por Benjamin Lee Whorf, los niveles metacomunicativos en el comportamiento social, así como la relatividad funcional de lenguas, estilos y géneros (Blommaert y Bulcaen, 2000: 460).

Posteriormente, Hidalgo Tenorio (2011) propone describir la heterogeneidad del Análisis Crítico del Discurso, su poder para atraer y no gustar, al mismo tiempo, y sus rasgos más interesantes y debilidades, que han causado debate y desacuerdo. La autora presta atención al CDA como investigación social orientada a los problemas y asentada sobre la historia social, la semiótica y la lingüística; y a los enfoques acadé- 
micos que también se consideran críticos con todas las objeciones planteadas contra el CDA. Como es de esperar, cita a Fairclough y a Blommaert y Bulcaen.

Dedica sus primeras líneas a resolver el primer obstáculo: definir qué es un discurso. Para ello, hace cinco definiciones (Hidalgo, 2011: 184-185):

- Discurso 1: Es la máxima unidad de descripción lingüística; fonemas, morfemas, palabras, frases, cláusulas, oraciones y textos están por debajo de él;

- Discurso 2: Es una muestra del uso del lenguaje, generalmente escrito para ser hablado, es decir, un discurso oral (speech);

- Discurso 3: Se refiere a la comunicación que se puede esperar en un contexto, situación, junto a un campo y registro, como el discurso del Derecho o la Medicina;

- Discurso 4: Es la interacción humana a través de cualquier medio, verbal y no verbal;

- Discurso 5: Se habla sólo como interacción;

- Discurso 6: Cubre todo el acto comunicativo.

Enmarca a Fairclough y a Wodak dentro del estudio del lenguaje en el habla y en la escritura, como parte del proceso social, y atendiendo al discurso como una forma de acción social que es socialmente constitutiva. Explica que Fairclough opta por la semiosis, a fin de no incluir solamente la comunicación lingüística, sino también la comunicación visual, generando muy diferentes significados. La semiosis juega un papel fundamental en la representación del mundo, acerca de cómo actuar, interactuar y construir la identidad, y puede ser identificada desde diferentes puntos de vista de los distintos grupos de actores sociales, tal como vaticinó Fairclough (Hidalgo, 2011: 186).

Más adelante, ahonda en el enfoque dialéctico-relacional de Fairclough sobre el CDA, como un marco esencialmente marxista, surgido de la investigación sobre el lenguaje, la ideología y el poder. Según Hidalgo (2011: 190), cuenta una terminología muy influyente, que se utilizará durante las décadas siguientes, con sintagmas como: dominio, resistencia, hibridación de las prácticas discursivas, tecnologización del discurso y conversacionalización del discurso público. Como Wodak y Meyer, Fairclough destaca la reflexión semiótica de los conflictos sociales en los discursos, y deja entrever su interés por los procesos sociales, es decir, las estructuras, las prácticas sociales y los acontecimientos. Asimismo, dice que Fairclough apoya la conciencia crítica del lenguaje, que para él es fundamental en la enseñanza de idiomas.

Como tercera fuente metodológica, acudimos al trabajo de Dunmire (2012). En su ensayo da una visión general de las investigaciones sobre el Critical Discourse Analysis (CDA) o Análisis Crítico del Discurso. Lo sitúa como uno de los cambios lingüísticos y políticos más importantes del final del siglo XX, dentro de las Ciencias Humanas y Sociales (Dunmire, 2012: 735). Se plantea diferentes concepciones de lo que comprende la Política y los objetos apropiados de estudio para el Análisis del Discurso Político (ADP). Cree que el ADP comprende la investigación inter y multidisciplinar, que se centra en la lingǘstica y en las dimensiones discursivas del texto político. Reconoce que el análisis del discurso no puede operar solamente dentro de 
un marco lingüístico y discursivo, sino que debe recurrir a métodos, marcos y contenidos de otras disciplinas para analizar adecuadamente su objeto de estudio. Es multidisciplinar porque reúne múltiples disciplinas para investigar temas socio-políticos y fenómenos que pertenecen a las diversas áreas de la erudición (Dunmire, 2012: 735).

Profundiza en los trabajos de Blommaert y Bulcaen, como una de las ramas más importantes e influyentes del estudio del discurso (Dunmire, 2012: 739). Expone que el programa de Blommaert legitima un enfoque social del análisis del discurso y aplaude su compromiso vinculando el análisis lingüístico con otros programas de investigación en Ciencias Sociales, para analizar las relaciones entre el poder, el lenguaje y los procesos sociales. Las debilidades del CDA, según Blommaert, residen en el fallo de producir interpretaciones sesgadas y restrictivas de los datos, para colapsar la semántica y la pragmática de la función textual (Dunmire, 2012: 740).

Por último, hemos acudido a otras fuentes teóricas que han analizado discursos y comunicación política, aplicados a diversos campos: Padilla y Semova (2009) observaron el discurso, los textos y los juegos psicológicos de las series de televisión favoritas de los universitarios madrileños; Valbuena (2010) ilustró el humor en la comunicación política y en los discursos políticos; Semova (2010) investigó los primeros cien días de gobierno búlgaro en los cuatro principales diarios del país; Peña Jiménez y Ortiz Sobrino (2011) asimilaron los eslóganes políticos españoles en las elecciones generales de 2008; Rodríguez Vidales (2012) defendió una interesante tesis doctoral sobre la Comunicación Política en la serie de televisión norteamericana El Ala Oeste de la Casa Blanca; Berrocal, Campos y Redondo (2012) disertaron sobre el infoentretenimiento político en YouTube; Valbuena y Padilla (2014) escribieron acerca de los debates políticos televisados, dedicando la primera parte de su texto a la retórica del discurso; Zamora, Berná y Martínez disertaron sobre la retórica emocional de la esposa del candidato, haciendo un análisis lingüístico de los discursos de Michelle Obama y Ann Romney; y Padilla (2014) ha reflexionado sobre la espectacularización del debate y del discurso electoral en Estados Unidos.

Con este armazón teórico y metodológico, procedemos a nuestro análisis del discurso del debate sobre el estado de la nación y lo sistematizamos en 3 interrogantes:

CI 1: Analizar ¿si los problemas más notables en la actualidad serán también los temas más tratados en los discursos de los líderes políticos del PP y PSOE?

CI 2: Según la clasificación de Fairclough, en el discurso del líder de la oposición sobre crisis-economía-empleo-social, ¿predominarán los argumentos tipo Opciones Alternativas (OA) o Premisas de Valor (PV).

CI 3: Según la clasificación de Fairclough, en el discurso del Presidente del Gobierno sobre crisis económica-empleo-social predominarán los argumentos tipo Premisas Circunstanciales (PC) y Premisas-Metas (PM).

\section{Desarrollo}

\subsection{Frecuencias}

Con el fin de conseguir un análisis de frecuencias viable, hemos excluido del recuento a las así denominadas "palabras vacías". Las tablas incluyen resultados iguales o superiores al $0,16 \%$ : 
Tabla 1. Frecuencia de palabras en los discursos de Rajoy y Rubalcaba.

Fuente: Elaboración propia, software NVivo

\begin{tabular}{|l|r|r|c|}
\cline { 2 - 4 } \multicolumn{1}{c|}{} & Longitud & Conteo & $\%$ \\
\hline España & 6 & 70 & 0,51 \\
\hline empleo & 6 & 48 & 0,35 \\
\hline reforma & 7 & 41 & 0,30 \\
\hline gobierno & 8 & 34 & 0,25 \\
\hline todos & 5 & 33 & 0,24 \\
\hline ley & 3 & 30 & 0,22 \\
\hline nuestro & 7 & 30 & 0,22 \\
\hline españoles & 9 & 27 & 0,20 \\
\hline medidas & 7 & 27 & 0,20 \\
\hline millones & 8 & 26 & 0,19 \\
\hline ahora & 5 & 24 & 0,18 \\
\hline empresas & 8 & 23 & 0,17 \\
\hline estado & 6 & 23 & 0,17 \\
\hline futuro & 6 & 23 & 0,17 \\
\hline crecimiento & 11 & 22 & 0,16 \\
\hline
\end{tabular}

Del análisis de frecuencias destaca el uso elevado de palabras como "España", "gobierno", "todos", "nuestro" y "españoles", algo que relacionamos directamente con el tono predominante en los debates sobre el estado de la nación en general. Los argumentos giran en torno de la cuestión de las reformas y el empleo. "Futuro" y "crecimiento" también están entre las palabras de uso frecuente durante el debate.

$\mathrm{Su}$ oponente, el líder socialista, no tiene ninguna respuesta a esta afirmación y de hecho, excluye por completo el tema del endeudamiento de su discurso. Otro error que comete Rubalcaba, surge del hecho de poner equivalencia entre el acto de autodeterminación y un referéndum de autodeterminación: "Tiene que quedar claro que el partido PSOE no va a votar a favor de la autodeterminación, pero a continuación decimos que queremos que este problema territorial debe resolverse y creemos que la fórmula es hacer una modificación pactada consensuada para que la Constitución reconozca la verdadera estructura federal del Estado y las singularidades de las comunidades y para que delimite las competencias del Estado y cambie el senado en cámara territorial".

El Presidente del Gobierno decide incluir el tema del referéndum de autodeterminación en el apartado de la "Defensa de la Constitución", lo que puede ser definido como una buena estrategia discursiva: "Señorías, quiero hacer referencia por último al anuncio de la futura convocatoria de un referéndum de autodeterminación en Cataluña. Un asunto de la máxima importancia política, como todos ustedes saben y que, por lo tanto, ha de ser de obligada consideración en este debate... es el conjunto del pueblo español quien tiene la capacidad para decidir lo que sea España. En esto consiste también la soberanía nacional. Por tanto, nadie, unilateralmente, puede privar al conjunto del pueblo español de su derecho a decidir sobre su futuro. Ni el Gobierno, ni ningún otro poder del Estado, ni siquiera esta cámara puede hacerlo. En consecuencia, señorías, como todos ustedes saben, ese referéndum no se puede celebrar. No es legal. Mi obli- 
gación es cumplir la ley y hacerla cumplir, lo que en este caso significa garantizar que nadie decida por el conjunto del pueblo español sobre el futuro de España".

Finalmente, el tema de la inmigración y los sucesos en Ceuta y Melilla apenas son tratados. Rubalcaba habla de "suceso dramático que le cuesta la vida a 15 personas" con las siguientes palabras: "Usted dice que la política de inmigración es política de Estado. No quiero volver al pasado. Una cosa es eso y otra, un suceso dramático que les cuesta la vida a 15 personas. Se trata de una actuación de algunos guardias civiles que no son defendibles. Tengo altísima estima por la Guardia Civil. Más que a ustedes les pese y les diré más, y ellos por mí. Altísima. Altísima y respeto y es así y por ello, por lo que creo que no debemos pasar lo que pasó porque los españoles tienen en la retina imágenes salvando náufragos y esto no es así y debe aclararse".

Rajoy, en cambio, habla de un problema que "la Europa comunitaria todavía no ha acertado a resolver": "Señorías, a raíz de las tragedias acaecidas frente a la costa de Lampedusa, el Consejo Europeo de octubre, sobre la base de propuestas presentadas por España, acordó la línea política para enfrentarse a las causas de los flujos migratorios. No necesito decirles nuestro interés sobre esta materia. Los recientes acontecimientos de Ceuta y Melilla ponen de actualidad un dramático problema que la Europa comunitaria todavía no ha acertado a resolver. Es preciso reformar la cooperación con los países de origen y tránsito, establecer una cooperación más estrecha con las organizaciones internacionales correspondientes y redoblar la lucha contra la trata de seres humanos y la inmigración clandestina, además de reforzar las actividades de la Agencia Frontex".

\subsection{Nodo "crisis"}

La palabra "crisis" ha sido pronunciada once veces en el discurso principal de Rajoy en el siguiente contexto: "Ni descansaré hasta que el peor rostro de la crisis económica, que es el paro, no comience a disminuir con fuerza" (PM), "el despido como único mecanismo de ajuste durante la crisis" (tesis-antitesis) (OP), "para hacer frente a la crisis" (D), "por primera vez en toda la crisis, señorías, podemos decir que hay menos parados que hace un año" (PMM), "pueda volver a producirse y, si se produce, que no pueda provocar las dramáticas consecuencias que ha desencadenado esta crisis" (PC), "aliviar cuanto sea posible las medidas que nos impuso el rigor de la crisis" (PC), "durante la crisis, entre 2007 y 2011 " (PC), "la crisis del euro no era sólo económica sino también política" (PC), "al salir de la crisis, España se muestre al mundo con un nuevo rostro, rejuvenecido" (PV). Excluimos de estos ejemplos las citas de titulares de periódicos de los distintos periódicos.

Rubalcaba utiliza 6 veces la palabra "crisis" en un discurso mucho más corto: 3.209 palabras frente a las 10.844 palabras de Rajoy, en el siguiente contexto: "la crisis como coartada" (PV), "nunca les gustó la negociación colectiva y la crisis ha servido para cargársela" (PV), "ha aprovechado la crisis para darle un golpe a la escuela pública" (PV), "la importancia de la UE para salir de la crisis económica" (PC), "hay una crisis política" (PC).

En resumen, podemos concluir que a la hora de referirse a la crisis, el Presidente del Gobierno recurre, en la mayoría de los casos, a premisas circunstanciales situando el problema dentro de un escenario de factores, sobre todo de carácter externo. Ru- 
balcaba limita su discurso, casi exclusivamente, a las premisas de valor que consisten en la expresión de emociones, juicios de valor y opinión.

Consulta de búsqueda de texto: vista preliminar de resultados

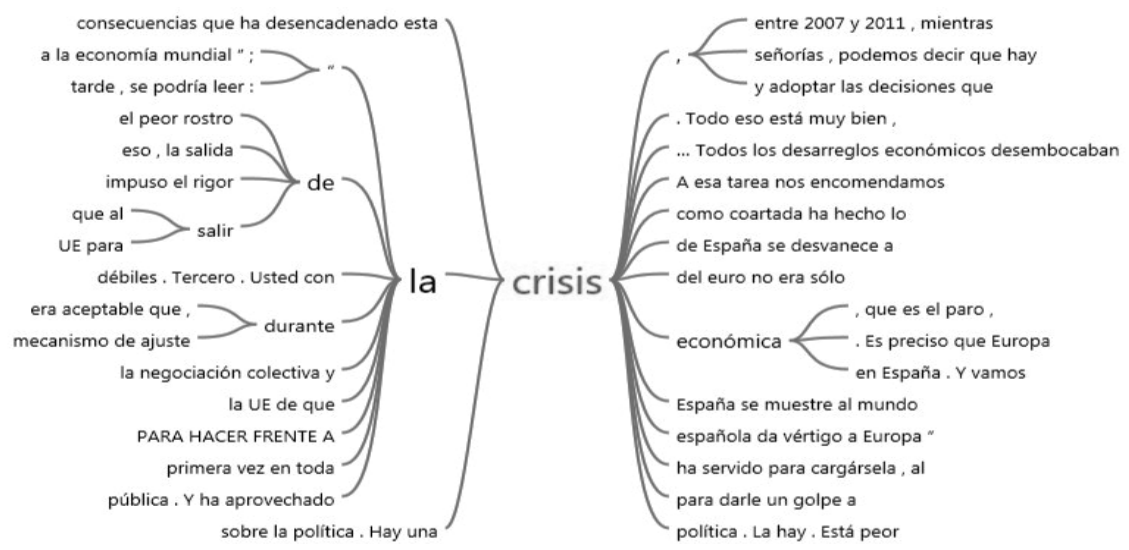

Figura 1. Nodo 1: La palabra "crisis" en los principales discursos pronunciados por Rajoy y Rubalcaba durante el debate del estado de la nación. Fuente: Elaboración propia, software NVivo

En resumen, en el discurso del líder socialista, la crisis es una coartada utilizada por el Gobierno para terminar con la negociación colectiva, para limitar la sanidad y la educación pública. En cambio, para su oponente, la crisis es, sobre todo, la causa de los altos índices de desempleo en el país. Por otra parte, ambos líderes reconocen la existencia de una crisis política. Por último, tanto Rajoy como Rubalcaba, sitúan la crisis en un entorno supranacional, europeo y mundial.

\subsection{Nodo "empleo"}

Hemos visto que la palabra "crisis" ha sido empleada un total de 17 veces, en los discursos principales de los líderes políticos del PP y PSOE. En cambio, la palabra "empleo" resulta ser la más utilizada (48 veces) después de "España" (70).

"Empleo" aparece 48 veces en el discurso de Rajoy y en la mayoría de los casos, en relación con los datos del paro. No obstante, tal y como podemos ver en los resultados, la frase más utilizada en este nodo ha sido "creación de empleo": "En este año 2014, se creará ya empleo neto, tanto en términos de contabilidad nacional, como en la Encuesta de Población Activa. Y contaremos también con más afiliados a la Seguridad Social. De hecho, ya tenemos, a día de hoy, más afiliados que en las mismas fechas de 2013".

Sorprende ver que en la mayoría de los casos (13), se trata de Premisas Valorativas (PV). Las Premisas de Medios/recursos y Metas (PMM) aparecen en 9 de los casos y las Premisas centradas en Metas (PM), sin mencionar la manera de llevarlas a cabo, en 5. En un número muy limitado de los casos (5), se hace referencia a la cuestión de cómo Abordar las Opciones Alternativas (AOA). Se trata de los ejemplos en los que Rajoy explica las nuevas medidas, experimentales en su carácter, de impulso al empleo. 
Rubalcaba recurre en 10 ocasiones a la palabra "empleo", en la mayoría de los casos, en referencia a los datos del paro. Traducido al esquema de los argumentos propuestos por Fairclough, en 6 de las 10 menciones, observamos Demanda (D) para encontrar una solución al problema descrito; en 3, Premisas de Valor (PV); y en una ocasión, Premisa-Meta (PM). Dos de las Premisas de Valor se caracterizan por su alta carga emocional: "No son mini jobs, son empleos basura", "les condena de por vida al subempleo".

En comparación, las Premisas Valorativas a las que recurre Rajoy, incluyen, entre otros, los siguientes argumentos: "Devolver a los parados el bienestar que proporciona un empleo", "la creación de empleo es el eje que vertebra todas nuestras iniciativas", "el empleo no es algo que pueda abordarse directamente", "hemos sido capaces de remover los obstáculos que impedían la creación de empleo", etc.

Consulta de búsqueda de texto: vista preliminar de resultados

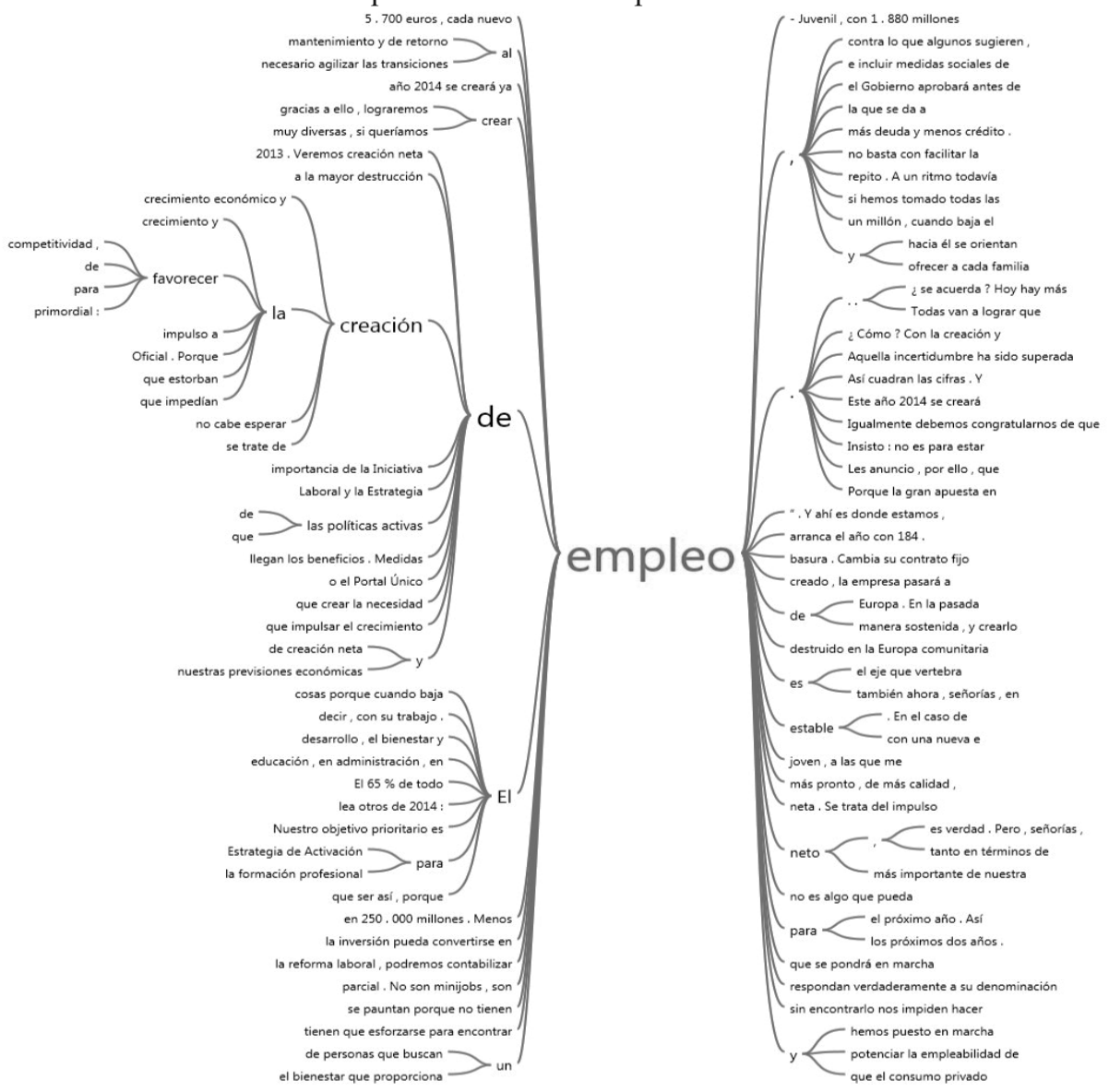

Figura 2. Nodo 2: La palabra "empleo" en los principales discursos pronunciados por Rajoy y Rubalcaba durante el debate del estado de la nación. Fuente: Elaboración propia, software NVivo 


\subsection{Nodo "economía"}

En el discurso de Rajoy, la palabra "economía" aparece 17 veces en el siguiente contexto: "Como nos demanda la situación de la economía" (PC), "la economía se hace más competitiva" (D), "la confianza en la economía española cayera bajo mínimos" (PC), "la economía española ha pasado de la recesión al crecimiento económico" (PM), "un diagnóstico correcto de los desequilibrios de la economía española" (D), "aquellos desequilibrios de la economía" (PC), "un ajuste estructural sin precedentes y sin comparación en las grandes economías avanzadas" (D), "la deuda de nuestra economía" (D), "recuperando la economía española en los mercados internacionales" (PM), "el Plan Estratégico de Internacionalización de la Economía Española 20142015" (PMM), "impulsar la competitividad de la economía española" (PM), "haya conseguido evitar un rescate global de nuestra economía" (OA), "economía digital" (OA), "economía europea" (PC). Excluimos, de nuevo, los titulares de los periódicos que recogen cómo Rajoy empieza su discurso.

En cambio, en el discurso de Rubalcaba la "economía" aparece como Premisa Circunstancial (PC) en una sola ocasión, en el siguiente contexto: "Europa abandona la recesión, toda. Y lo hace porque Draghi dijo lo que dijo. Aviso a navegantes. Porque Merkel dijo que Grecia no se iba y estabilizó la zona euro y porque la Comisión dejó la política de ajustes que nos mataba. Por estas tres razones, la economía española y la griega y la irlandesa y la portuguesa están mejor".

En resumen, podemos decir que tanto el Gobierno como la oposición, a la hora de hablar de la economía, prefieren centrarse en premisas circunstanciales, es decir, argumentos que vinculan la situación en el país directamente con el entorno europeo e internacional o con la herencia del anterior gobierno. Otro argumento empleado con la misma frecuencia es la demanda de soluciones que se caracteriza por la mera descripción del problema y por la ausencia de metas y medidas concretas:

Consulta de búsqueda de texto: vista preliminar de resultados

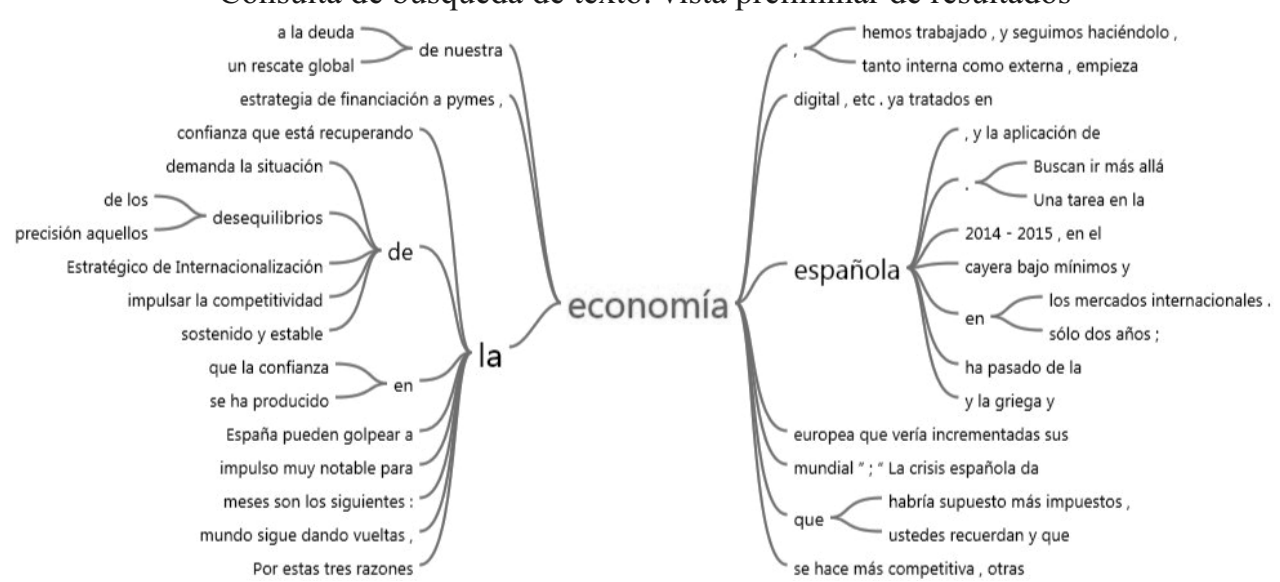

Figura 3. Nodo 3: La palabra "economía" en los principales discursos pronunciados por Rajoy y Rubalcaba durante el debate del estado de la nación. Fuente: Elaboración propia, software NVivo 
Esta puede ser una buena estrategia por parte del Partido Popular pero no en el caso del Partido Socialista. Al recurrir a premisas circunstanciales, Rubalcaba disminuye el impacto de lo que debería ser uno de sus argumentos centrales.

\subsection{Nodo "social"}

La palabra "social" aparece en la mayoría de los casos, en relación con afiliación, cotización y alta, es decir, con el sistema de la Seguridad Social. En el discurso de Rajoy, el concepto aparece un total de 19 veces, mientras en el caso de su oponente político, solamente 5 .

El Presidente del Gobierno habla de: "gasto social", "servicios sociales", "agentes económicos y sociales", "Seguridad Social" (9 veces), "protección social", "medidas sociales de apoyo", "cohesión social", "reducción de las cargas sociales", "sectores sociales en difícil situación", "capital social" y "gastos sociales extraordinarios". Rubalcaba se centra en los siguientes aspectos: "gasto social" (D), "concertaciones sociales" (D, 2 veces), "Seguridad Social" (AOA) y "conquistas sociales" (PV). De nuevo, podemos decir que el líder socialista desaprovecha los puntos fuertes de la ideología de su partido. En concreto, los relacionados con la protección social, debido a los pocos aspectos que menciona a la hora de referirse al tema y, también, por poner el acento sobre las concertaciones sociales, un punto, quizás, demasiado abstracto y no del todo claro para audiencia.

Consulta de búsqueda de texto: vista preliminar de resultados

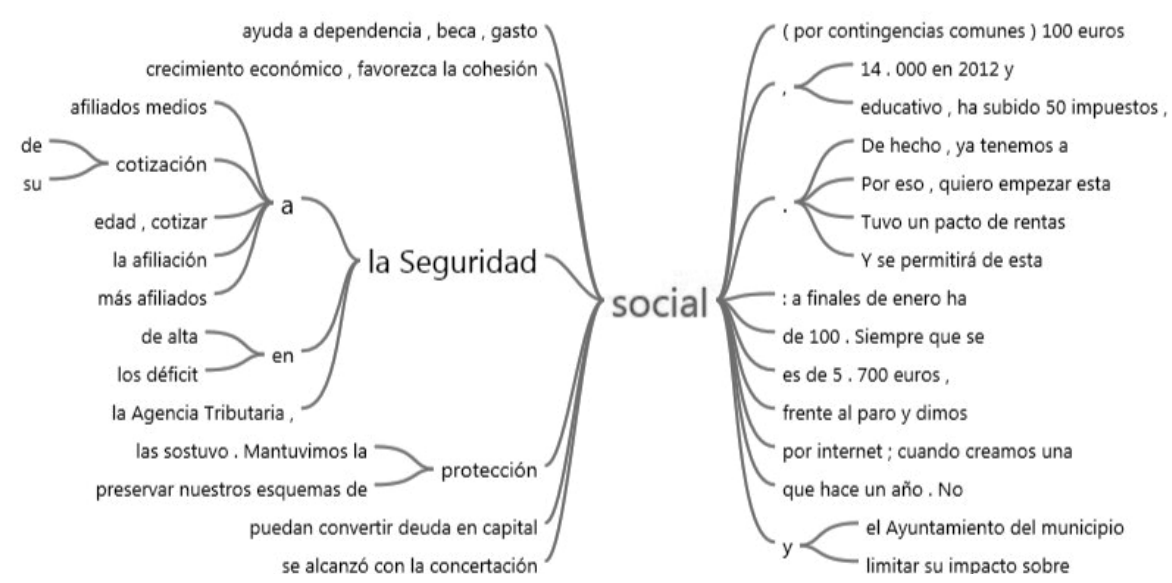

Figura 4. Nodo 4: La palabra "social" en los principales discursos pronunciados por

Rajoy y Rubalcaba durante el debate del estado de la nación. Fuente: Elaboración propia, software NVivo

En cambio, la mayor parte de los argumentos en el discurso del partido gobernante se refieren a la manera de abordar las opciones alternativas ( $7 \mathrm{AOA}$ ), seguidos por la premisas de recursos y metas (4 PMM). El resto se reparte como sigue: Demanda (D): 3, Premisas Circunstanciales (PC): 1, Premisas de Valor (PV): 3, Opciones Alternativas $(\mathrm{OA}): 1$. 
La propuesta de opciones alternativas en una situación de crisis, suele dar buenos resultados debido al alto impacto en la audiencia. No obstante, ambos partidos fallan a la hora de dar suficientes alternativas de este tipo, lo que consideramos, puede ser, el punto más débil del debate sobre el estado de la nación de 2014.

\section{Conclusiones}

El debate sobre el estado de la nación se celebra una vez al año, en el Congreso de los Diputados de España, y aborda, durante una o dos jornadas, toda la política general llevada a cabo por el Gobierno, durante el último año. No aparece como celebración o evento obligatorio en la Constitución Española, pero se ha establecido como una práctica parlamentaria anual ineludible desde el año 1983.

En este artículo, hemos analizado, concretamente, el debate sobre el estado de la nación del año 2014, que se desarrolló durante dos jornadas: el martes 25 de febrero y el miércoles 26 de febrero. Codificamos los discursos del presidente de Gobierno, Mariano Rajoy (Partido Popular), y el entonces líder de la oposición, Alfredo Pérez Rubalcaba (Partido Socialista Obrero Español), extrayendo nubes de palabras, mapas ramificados y árboles de palabras que han mostrado los conceptos más frecuentes. Mediante la creación de nodos y su correspondiente codificación, hemos conseguido categorizar las ideas y, también, organizar las mismas por conceptos previamente elegidos: "Crisis", "Economía", "Empleo" y "Social". Asimismo, hemos analizado el discurso desde el planteamiento propuesto por Isabela y Norman Fairclough (2012), que nace a raíz del análisis del discurso del Partido Liberal y del Primer Ministro, Tony Blair.

Del análisis de frecuencias destaca el uso elevado de palabras como "España", "gobierno", "todos", "nuestro" y "españoles", algo que relacionamos directamente con el tono predominante en los debates sobre el estado de la nación en general. Los argumentos giran en torno de la cuestión de las reformas y el empleo. "Futuro" y "crecimiento" también están entre las palabras de uso frecuente durante el debate. Por otro lado, las palabras menos frecuentes son: "aborto", "ajustes", "autodeterminación", "dependencia", "endeudamiento", "fraude", "inmigración", "información", "inversiones", "investigación" y "liquidez".

Analizando los nodos, en el discurso del líder socialista, la crisis es una coartada utilizada por el Gobierno para terminar con la negociación colectiva, para limitar la sanidad y la educación pública. En cambio, para su oponente, la crisis es, sobre todo, la causa de los altos índices de desempleo en el país. El nodo "Empleo" aparece 48 veces en el discurso de Rajoy y en la mayoría de los casos, en relación con los datos del paro. Sin embargo, la frase más utilizada en este nodo ha sido "creación de empleo". El tercer nodo, "economía", aparece 17 veces en contextos positivos: "Como nos demanda la situación de la economía", "la economía se hace más competitiva", "la confianza en la economía española cayera bajo mínimos", "la economía española ha pasado de la recesión al crecimiento económico", "un diagnóstico correcto de los desequilibrios de la economía española", "recuperando la economía española en los mercados internacionales" o "impulsar la competitividad de la economía española. El 
cuarto nodo, "social", aparece en relación con afiliación, cotización y alta, es decir, con el sistema de la Seguridad Social. En el discurso de Rajoy, el concepto aparece un total de 19 veces, mientras en el caso de su oponente político, solamente 5 .

Así, el debate sobre el estado de la nación se convierte en un momento que resalta la democracia parlamentaria en el Congreso de los Diputados, donde las tensiones entre los grupos de la oposición y el jefe de gobierno juegan en un escenario controlado por la solemnidad del protocolo. En este sentido, Mariano Rajoy y Alfredo Pérez Rubalcaba, se posicionaron en plataformas opuestas de procesamiento y observación de los datos para discutir.

Pese al calibre de subjetividad que connota la categoría de "interpretación", en el caso de este análisis del discurso responde a un marco objetivo con claves que han sido posible detectar a través del software NVivo, una herramienta informática que otorga pertinencia científica a nuestra investigación puesto que logramos secuencias concretas de palabras con las que es posible trazar la estructura de los discursos y los encuadres que los soportan. Confiamos en que la metodología empleada sirve de prospectiva de investigación para futuros estudios académicos y análisis del discurso.

\section{Referencias bibliográficas}

BERROCAL, Salomé; CAMPOS, Eva; y REDONDO, Marta (2012): “Comunicación Política en Internet: la tendencia al infoentretenimiento político en YouTube". Estudios sobre el Mensaje Periodístico, vol. 18, núm. 2, pp. 643-659. Madrid, Servicio de Publicaciones de la Universidad Complutense. DOI: http://dx.doi.org /10.5209/rev_ESMP.2012.v18.n2.41037

BLOMMAERT, Jan and BULCAEN, Chris (2000): "Critical Discourse Analysis". Annual Review of Anthropology, num. 29. Palo Alto, Annual Reviews, pp. 447-466.

DUNMIRE, Patricia L. (2012): "Political Discourse Analysis: Exploring the Language of Politics and the Politics of Language". Language and Linguistics Compass, num. 6, Issue 11, Sociolinguistics. Nueva Jersey, John Wiley \& Sons, pp. 735-751.

FAIRCLOUGH, Isabela and FAIRCLOUGH, Norman (2012): Political Discourse Analysis: A Method for Advanced Students. Londres, Routledge.

HIDALGO TENORIO, Encarnación (2011): "Critical Discourse Analysis, An overview". Nordic Journal of English Studies, Vol. 10, num. 1. Nordic Association of English Studies, pp. 183-210.

JIVKOVA SEMOVA, Dimitrina (2010): "Los primeros cien días del nuevo gobierno de Bulgaria según la prensa: un análisis de contenido". CIC: Cuadernos de Información y Comunicación, vol. 15, pp. 251-261. Madrid, Servicio de Publicaciones de la Universidad Complutense.

PADILLA, Graciela (2014): "La espectacularización del debate electoral: estudio del caso en Estados Unidos". Revista de Comunicación Vivat Academia, Septiembre, núm. 128, pp. 107-123. DOI: http://dx.doi.org/10.15178/va.2014.128.107-123. En: http://www.vivatacademia.net/index.php/vivat/article/view/587. 
PADILLA, Graciela y JIVKOVA SEMOVA, Dimitrina (2009): "Los juegos de las series favoritas de los universitarios madrileños". CIC: Cuadernos de Información y Comunicación, vol. 14, pp. 199-211. Madrid, Servicio de Publicaciones de la Universidad Complutense.

PEÑA JIMÉNEZ, Palma y ORTIZ SOBRINO, Miguel Ángel (2011): "El eslogan político español en la campaña de elecciones generales de 2008". Estudios sobre el Mensaje Periodístico, vol. 17, núm. 2, pp. 549-568. Madrid, Servicio de Publicaciones de la Universidad Complutense. DOI: http://dx.doi.org/10.5209 /rev_ESMP.2011.v17.n2.38130

RODRÍGUEZ VIDALES, Yolanda (2012): El Ala Oeste de la Casa Blanca y otros modelos de ficción para comprender la Comunicación Política. Madrid, Universidad Complutense de Madrid. Disponible en: http://eprints.ucm.es/17551 /1/T34094.pdf [Consulta: 30 de septiembre de 2014].

VALBUENA, Felicísimo (2010): "El humor en la Comunicación Política". CIC: Cuadernos de Información y Comunicación, vol. 15, pp. 165-185. Madrid, Servicio de Publicaciones de la Universidad Complutense.

VALBUENA, Felicísimo y PADILLA, Graciela (2014): "Los debates políticos televisados", en HERRERO, Julio César y RÖHMER, Max: Comunicación en campaña. Dirección de campañas electorales y marketing político. Madrid, Pearson, pp. 271-302.

ZAMORA, Rocío; BERNÁ, Celia; y MARTÍNEZ, Helena (2014): "La retórica emocional de la esposa del candidato: análisis lingüístico de los discursos de Michelle Obama y Ann Romney". Estudios sobre el Mensaje Periodístico, volumen 20, núm. 1, pp. pp. 549-568. Madrid, Servicio de Publicaciones de la Universidad Complutense. DOI: http://dx.doi.org/10.5209/rev_ESMP.2014.v20.n1.45250

Dimitrina Jivkova Semova es Profesora Asociada del Departamento de Periodismo III, Facultad de Ciencias de la Información de la Universidad Complutense de Madrid.

Graciela Padilla Castillo es Profesora Titular interina del Departamento de Periodismo III, Facultad de Ciencias de la Información de la Universidad Complutense de Madrid.

Noé Pernía Peñalver es investigador doctor del Departamento de Periodismo I, Facultad de Ciencias de la Información de la Universidad Complutense de Madrid. 